\title{
Study of the dynamics of third-order iterative methods on quadratic polynomials *
}

\author{
Alicia Cordero, Juan R. Torregrosa * \\ Instituto de Matemática Multidisciplinar, \\ Universitat Politècnica de València, Camino de Vera, s/n, 46022 \\ Valencia, Spain \\ Pura Vindel, \\ Instituto de Matemáticas y Aplicaciones de Castellón \\ Universitat Jaume I, Av. de Vicent Sos Baynat s/n, 12071 \\ Castellón de la Plana, Spain
}

\begin{abstract}
In this paper we analyze the dynamical behaviour of the operators associated to multi-point iterative methods and frozen derivative methods, for solving nonlinear equations, applied on second degree complex polynomials. We obtain that, in both cases, the Julia set is a connected set that separates the basins of attraction of the roots of the polynomial. Moreover, the Julia set of the operator associated to multi-point iteration methods is the same as the Newton operator, although it is more complicated for the frozen derivative operator. We explain these differences by obtaining the conjugacy function of each method and by showing that the operators associated to Newton's method and multi-point iteration methods are both conjugate to powers of $z$.
\end{abstract}

Key words: Nonlinear equations, iterative methods, complex dynamics, conjugacy map, basin of attraction

‡ This research was supported by Ministerio de Ciencia y Tecnología MTM201018539 and by Vicerrectorado de Invetigación, Universitat Politècnica de València PAID-06-2010-2285

* Correspondig author

Email addresses: acordero@mat.upv.es (Alicia Cordero), jrtorre@mat.upv.es (Juan R. Torregrosa ), vindel@uji.es (Pura Vindel). 


\section{Introduction}

Many engineering applications involve nonlinear equations $f(x)=0$ whose solution can not be found by means of analytical methods. To approximate the solution of these equations we use iterative methods. This means that the output of the method is a sequence of images $\left\{x_{0}, R\left(x_{0}\right), R^{2}\left(x_{0}\right), \ldots, R^{n}\left(x_{0}\right), \ldots\right\}$ for the initial condition $x_{0}$, where $R$ is a rational function that represents the fixed point operator of the iterative scheme. Therefore, it can be seen as a discrete dynamical system and we can study it from this point of view.

There is an extensive literature on the study of iteration of rational mappings $R$ of a complex variable (see [1], [2], for example) and, as it is well known, the Newton's method (see [3], [4] for example) applied on polynomials is a rational function. In this case, the Riemann sphere $\mathbb{C}$ is also considered as the domain of the rational mapping $R$ associated to the iterative method.

To our knowledge, the study on the dynamics of Newton's method has been extended to other point-to-point iterative methods used for solving nonlinear equations, with convergence order up to three (see, for example [5], [6] and, more recently, [7] and [8]).

S. Amat et al. in [9] make a brief raid into the study of the dynamics of the Potra-Pták method (see [10]) defined on the real numbers and applied on polynomials of second and third degrees. Nevertheless, this study, interesting in itself, does not allow to see all the richness of the dynamics of the method when it is defined on the complex numbers.

Now, let us recall some basic concepts on complex dynamics. Given a rational function $R: \hat{\mathbb{C}} \rightarrow \hat{\mathbb{C}}$. The orbit of a point $z_{0} \in \hat{\mathbb{C}}$ is defined as:

$$
z_{0}, R\left(z_{0}\right), R^{2}\left(z_{0}\right), \ldots, R^{n}\left(z_{0}\right), \ldots
$$

and we are interested in the study of the asymptotic behaviour of the orbits depending on the initial condition $z_{0}$, that is, we are interested in the study of the phase plane of the map defined by the iterative method.

To obtain this phase space, the first of all is to classify the initial conditions from the asymptotic behavior of their orbits.

Let us consider $\alpha \in \mathbb{C}$ a root of $f, f(\alpha)=0$. The basin of attraction of $\alpha$ is defined as the set of pre-images of any order:

$$
\mathcal{A}(\alpha)=\left\{z_{0} \in \hat{\mathbb{C}}: R^{n}\left(z_{0}\right) \rightarrow \alpha, n \rightarrow \infty\right\} .
$$

A $z_{0} \in \hat{\mathbb{C}}$ is called a fixed point if it satisfies: $R\left(z_{0}\right)=z_{0}$. A periodic point $z_{0}$ 
of period $p>1$ is a point such that $R^{p}\left(z_{0}\right)=z_{0}$ and $R^{k}\left(z_{0}\right) \neq z_{0}, k<p$. A pre-periodic point is a point $z_{0}$ that is not periodic but there exists a $k>0$ such that $R^{k}\left(z_{0}\right)$ is periodic. A critical point $z_{0}$ is a point where the derivative of rational function vanishes, $R^{\prime}\left(z_{0}\right)=0$.

On the other hand, a fixed point $z_{0}$ is called attractor if $\left|R^{\prime}\left(z_{0}\right)\right|<1$, superattractor if $\left|R^{\prime}\left(z_{0}\right)\right|=0$, and repulsor if $\left|R^{\prime}\left(z_{0}\right)\right|>1$.

The set of points $z \in \hat{\mathbb{C}}$ such that their families $\left\{R^{n}(z)\right\}_{n \in \mathbb{N}}$ are normal in some neighborhood $U(z)$, is the Fatou set, $\mathcal{F}(R)$, that is, the Fatou set is composed by the set of points whose orbits tend to an attractor (fixed point, periodic orbit or infinity). Its complement in $\hat{\mathbb{C}}$ is the Julia set, $\mathcal{J}(R)$; therefore, the Julia set includes all repelling fixed points, periodic orbits and their pre-images. That means that the basin of attraction of any fixed point belongs to the Fatou set. On the contrary, the boundaries of the basins of attraction belong to the Julia set.

\subsection{The Newton's Method}

The Newton's method is the best known method to find the roots of a nonlinear function:

$$
f(z)=0,
$$

where $f \in C^{1}(\hat{\mathbb{C}})$ is defined on the Riemann sphere $\hat{\mathbb{C}}$. The Newton's iterative operator is

$$
N_{f}(z)=z-\frac{f(z)}{f^{\prime}(z)}
$$

which satisfies that $f(z)=0$ if and only if $N_{f}(z)=z$. So, to find the roots of $f(z)$ is equivalent to find the fixed points of the operator $N_{f}(z)$. Actually, the global analysis of convergence of Newton's method on $f(z)$ is equivalent to compute individual orbits of the dynamical systems generated by the Newton's $\operatorname{map} N_{f}(z)$.

The equation (1) on a polynomial $p(z)$

$$
N_{p}(z)=z-\frac{p(z)}{p^{\prime}(z)}
$$

verifies the following properties:

1. The roots of $p(z)$ correspond to the finite fixed points of $N_{p}$.

2. The point at the infinity is a repelling fixed point.

3. As the derivative of the iteration function is

$$
N_{p}^{\prime}(z)=\frac{p(z) p^{\prime \prime}(z)}{p^{\prime}(z)^{2}}
$$


the simple roots of $p(z)$ are superattracting fixed points. Multiple roots are attracting fixed points, but not superattracting.

For simplicity, we begin studying the Newton's method on quadratic polynomials. It is known that the roots of the polynomial can be transformed by an affine map without qualitatively changing the dynamics of the Newton'iteration function. So, we can use the quadratic polynomial $p(z)=z^{2}+c$.

Then, we obtain that the two basin of attraction of the roots are separated by the perpendicular bisector of the line segment from one root to the other. This bisector is the Julia set for this polynomial, that it is connected.

Indeed, it is desirable that the convergence regions of both maps be essentially the same, except for the change of coordinates, see [11] for example.

Theorem 1 Let $f$ be an analytic function on the Riemann sphere, and let $A(z)=\alpha z+\beta$, with $\alpha \neq 0$, be an affine map. If $g(z)=\lambda(f \circ A)(z)$, where $\lambda \in \mathbb{C}-\{0\}$, then the Newton's iteration function $N_{f}$ is analytically conjugated to $N_{g}$ by $A$ :

$$
A \circ N_{f} \circ A^{-1}(z)=N_{g}(z) .
$$

Moreover,

Theorem 2 [3, Th. 2] Let $p(z)$ be a quadratic polynomial with distinct roots. The Newton's operator $N_{p}(z)$ is globally, analytically conjugate to the quadratic polynomial $z^{2}$.

P. Blanchard, in [3], proves it by considering the conjugacy map

$$
h(z)=\frac{z-i \sqrt{c}}{z+i \sqrt{c}}
$$

with the following properties:

i) $h(\infty)=1$,

ii) $h(i \sqrt{c})=0$,

iii) $h(-i \sqrt{c})=\infty$.

Then,

$$
\left(h \circ N_{p} \circ h^{-1}\right)(z)=z^{2} .
$$

So, for quadratic polynomials, the Newton's operator is always conjugate to the rational map $z^{2}$, satisfying the following properties:

1. The dynamics of this operator gives the unit circle $S^{1}(z)=\{z \in \hat{\mathbb{C}}:|z|=$ $1\}$ as the invariant Julia set. 
2. The Fatou set is defined by the two basins of attraction of the superattracting fixed points: 0 and $\infty$.

In this paper, we study the dynamics of two families of iterative methods of order three: the multi-point interpolation methods (Section 2) and the frozen derivative methods (Section 3) on quadratic polynomials defined on the complex plane.

\section{The Multi-Point Interpolation Methods}

From quadrature formulas applied to the integral

$$
f(x)=f\left(x_{k}\right)+\int_{x_{k}}^{x} f^{\prime}(t) d t
$$

some authors (see [12] and [13]) have derived a family of multi-point interpolation methods, whose iterative scheme is

$$
x_{k+1}=x_{k}-\frac{f\left(x_{k}\right)}{\sum_{j=1}^{m} A_{j} f^{\prime}\left(\eta_{j}\left(x_{k}\right)\right)},
$$

where $\eta_{j}\left(x_{k}\right)=x_{k}-\tau_{j} \frac{f\left(x_{k}\right)}{f^{\prime}\left(x_{k}\right)}, j=1,2, \ldots, m$, with $\tau_{j}$ the knots in $[0,1]$ and $A_{j}$ the weight of the interpolatory quadrature formula used. So, they satisfy the relationships:

$$
\sum_{j=1}^{m} A_{j}=1
$$

and

$$
\sum_{j=1}^{m} A_{j}\left(1-\tau_{j}\right)=\frac{1}{2} .
$$

These identities alow the authors assure that the resulting methods have, at least, order of convergence three.

We also state the Scaling Theorem for this iterative scheme, whose fixed point operator is denoted by $M_{f}$.

Theorem 3 Let $f$ be an analytic function on the Riemann sphere, and let $A(z)=\alpha z+\beta$, with $\alpha \neq 0$, be an affine map. If $g(z)=\lambda(f \circ A)(z)$, where $\lambda \in \mathbb{C}-\{0\}$, then the fixed point operator $M_{f}$ is analytically conjugated to $M_{g}$ by $A$ :

$$
A \circ M_{f} \circ A^{-1}(z)=M_{g}(z) .
$$


We study the dynamics of the operator of this family of iterative methods on quadratic polynomial $p(z)=z^{2}+c, c \in \mathbb{C}$.

$$
\begin{aligned}
M_{p}(z)= & z-\frac{f(z)}{\sum_{j=1}^{m} A_{j} f^{\prime}\left(\eta_{j}(z)\right)}=z-\frac{\left(z^{2}+c\right) z}{-\sum_{j=1}^{m} A_{j}\left(c \tau_{j}-2 z^{2}+\tau_{j} z^{2}\right)}= \\
= & \frac{-z^{3}+c z+\left(c z+z^{3}\right) \sum_{j=1}^{m} A_{j} \tau_{j}}{-2 z^{2}+\left(z^{2}+c\right) \sum_{j=1}^{m} A_{j} \tau_{j}}
\end{aligned}
$$

and, by using (7) and (8), we obtain:

$$
M_{p}(z)=\frac{3 c z-z^{3}}{c-3 z^{3}}
$$

The fixed points of $M_{p}(z)$ are the roots of the polynomial:

$$
M_{p}(z)=z \Rightarrow z= \pm i \sqrt{c}
$$

and there are also the critical points:

$$
M_{p}^{\prime}(z)=\frac{3\left(z^{2}+c\right)^{2}}{\left(c-3 z^{2}\right)^{2}}=0 .
$$

As in the previous cases, these roots are superattractor fixed points.

Therefore, similarly to the Newton method, the Fatou set consists of the basins of attraction of the two roots of the polynomial. That means that this method never fail on quadratic polynomials when it is applied on open set of the complex plane. The dynamical plane of the operator (9) is the same as the Newton method. This result is very well understood from the following theorem.

Theorem 4 Let $p(z)$ be a quadratic polynomial with distinct roots. The fixed point operator $M_{p}(z)$ associated to the family of iterative methods described in (6) has the following properties:

i) $M_{p}(z)$ is globally, analytically conjugate to the cubic polynomial $z^{3}$.

ii) The dynamics of this operator gives the unit circle $S^{1}(z)=\{z \in \hat{\mathbb{C}}:|z|=$ $1\}$ as the invariant Julia set. Moreover, this set is connected.

iii) The Fatou set is defined by the two basins of attraction of the superattracting fixed points: 0 and $\infty$. 
Proof: We consider the conjugacy map:

$$
h(z)=\frac{z-i \sqrt{c}}{z+i \sqrt{c}}
$$

which has the same properties as in the previous theorem: $h(\infty)=1, h(i \sqrt{c})=$ 0 and $h(-i \sqrt{c})=\infty$. So,

$$
h^{-1}(z)=i \sqrt{c} \frac{z+1}{1-z}
$$

and, therefore

$$
\left(h \circ M_{p} \circ h^{-1}\right)(z)=z^{3}
$$

is a cubic polynomial of degree three that has superattracting fixed points at 0 and $\infty$ separated by the unit circle. As this map does not depend on the parameter $c$, the Julia set is the unit circle and, therefore, it is connected for every $c$.

Moreover, (11) implies that the origin is a zero of order three.

\subsection{Iterative Methods coming from operator $M_{f}$}

There are some well known iterative methods that come from this operator, among others: the Midpoint Method, the Trapezoidal method and the Simpson Method.

- The Midpoint Method was developed by Özban in [14] and can be written as

$$
x_{k+1}=x_{k}-\frac{f\left(x_{k}\right)}{f^{\prime}\left(\frac{x_{k}+y_{k}}{2}\right)},
$$

where $y_{k}$ is the Newton's iteration. The corresponding operator is:

$$
M_{f}(z)=z-\frac{f(z)}{f^{\prime}\left(z-\frac{f(z)}{2 f^{\prime}(z)}\right)} .
$$

- Similarly, Frontini and Sormani derived in [13] the Trapezoidal Method

$$
x_{k+1}=x_{k}-\frac{2 f\left(x_{k}\right)}{f^{\prime}\left(x_{k}\right)+f^{\prime}\left(y_{k}\right)},
$$

yields to the operator

$$
\operatorname{Tr}_{f}(z)=z-\frac{2 f(z)}{f^{\prime}(z)+f^{\prime}\left(z-\frac{f(z)}{f^{\prime}(z)}\right)}
$$


- Finally, Frontini and Sormani also presented in [13] the Simpson Method:

$$
x_{k+1}=x_{k}-\frac{6 f\left(x_{k}\right)}{f^{\prime}\left(x_{k}\right)+4 f^{\prime}\left(\frac{x_{k}+y_{k}}{2}\right)+f^{\prime}\left(y_{k}\right)}
$$

provides the operator

$$
S_{f}(z)=z-\frac{6 f(z)}{f^{\prime}(z)+4 f^{\prime}\left(z-\frac{f(z)}{2 f^{\prime}(z)}\right)+f^{\prime}\left(z-\frac{f(z)}{f^{\prime}(z)}\right)} .
$$

As we have seen these three operators $(12),(13)$ and (14) acting on the family of quadratic polynomial $p(z)=z^{2}+c, c \in \mathbb{C}$ give the operator $M_{p}(z)=$ $\frac{3 c z-z^{3}}{c-3 z^{3}}=S_{p}(z)=\operatorname{Tr}_{p}(z)$ Consequently, their dynamics have been studied in the previous section. Therefore,

Remark The dynamics of the Midpoint, Trapezoidal and Simpson methods on quadratic polynomial defined on the complex plane is the same as the Newton method.

\section{The Frozen Derivative Methods}

In this section we study the dynamics of a family of multi-point iterative methods obtained from Newton's method by replacing $f(z)$ by a linear combination of values of $f(z)$ in different points. Specifically, the general scheme is

$$
\begin{gathered}
x_{k+1}=x_{k}-\frac{\sum_{j=1}^{m} B_{j} f\left(\eta_{j}\left(x_{k}\right)\right)}{f^{\prime}\left(x_{k}\right)} \\
\eta_{j}\left(x_{k}\right)=x_{k}-\tau_{j} \frac{f\left(x_{k}\right)}{f^{\prime}\left(x_{k}\right)}, j=1,2, \ldots, m
\end{gathered}
$$

where $\tau_{j}$ and $B_{j}$ are parameters to be chosen in $[0,1]$ and $\mathbb{R}$ respectively, and $m$ is a positive integer (see [15]). These parameters satisfy the following relationships:

$$
\sum_{j=1}^{m} B_{j}\left(1-\tau_{j}\right)=1, \quad \sum_{j=1}^{m} B_{j} \tau_{j}^{2}=1 .
$$

The value of these parameters yields to different methods, as we will see in the following; moreover, they play an important role in the order of convergence of the method.

The fixed point operator of these methods is: 


$$
O_{p}(z)=z-\frac{\sum_{j=1}^{m} B_{j} f\left(\eta_{j}(z)\right)}{f^{\prime}(z)},
$$

where $\eta_{j}(z)=z_{k}-\tau_{j} \frac{f\left(z_{k}\right)}{f^{\prime}\left(z_{k}\right)}$ and the parameters $\tau_{j}$ and $B_{j}$ satisfy (16).

The Scaling Theorem for this operator can be established in the following way.

Theorem 5 Let $f$ be an analytic function on the Riemann sphere, and let $A(z)=\alpha z+\beta$, with $\alpha \neq 0$, be an affine map. If $g(z)=\lambda(f \circ A)(z)$, where $\lambda \in \mathbb{C}-\{0\}$, then the fixed point operator $O_{f}$ is analytically conjugated to $O_{g}$ by $A$ :

$$
A \circ O_{f} \circ A^{-1}(z)=O_{g}(z) .
$$

Now, we are going to study its dynamics for quadratic polynomial defined on the complex plane, $p(z)=z^{2}+c, c \in \mathbb{C}$.

$$
\begin{aligned}
O_{p}(z) & =z-\frac{\sum_{j=1}^{m} B_{j} f\left(\eta_{j}(z)\right)}{f^{\prime}(z)}= \\
= & z-\frac{\sum_{j=1}^{m} B_{j}\left(c^{2} \tau_{j}^{2}+4 c z^{2}-4 c \tau_{j} z^{2}+2 c \tau_{j}^{2} z^{2}+4 z^{4}-4 \tau_{j} z^{4}+\tau_{j}^{2} z^{4}\right)}{8 z^{3}}= \\
= & \frac{8 z^{4}-\left(c^{2}+2 c z^{2}+z^{4}\right) \sum_{j=1}^{m} B_{j} \tau_{j}^{2}-\left(4 c z^{2}+4 z^{4}\right) \sum_{j=1}^{m} B_{j}\left(1-\tau_{j}\right)}{8 z^{3}} .
\end{aligned}
$$

By using the relationships (16) between the parameters, we obtain a common expression of the operator for any member of the family (15):

$$
O_{p}(z)=\frac{3 z^{4}-6 c z^{2}-c^{2}}{8 z^{3}}
$$

This operator has four fixed points: two of them are the roots of the polynomial. The other two are called strange fixed points.

$$
O_{p}(z)=z \Rightarrow z= \pm i \sqrt{c}, \pm i \sqrt{\frac{c}{5}}
$$

The dynamical properties of a complex analytical functions are often determined for the dynamics of his critical points. In this case, the derivative of 
$(18)$

$$
O_{p}^{\prime}(z)=\frac{3}{8} \frac{\left(z^{2}+c\right)^{2}}{z^{4}},
$$

allows us to deduce that the only critical points are the roots of the polynomial. Moreover,

$$
O_{p}^{\prime}( \pm i \sqrt{c})=0
$$

implies that these roots are superattractor critical points. The other roots of $O_{p}(z)$ are repulsive fixed points $\left(O_{p}^{\prime}\left( \pm i \sqrt{\frac{c}{5}}\right)=6\right)$; so, they are in the Julia set.

As in Newton's method, the Fatou set consists of the basins of attraction of the two roots of the polynomial. That means that this method never fails for quadratic polynomials when it is applied on open set of the complex plane. The dynamical plane of the operator (18) is shown in the Figure 1.

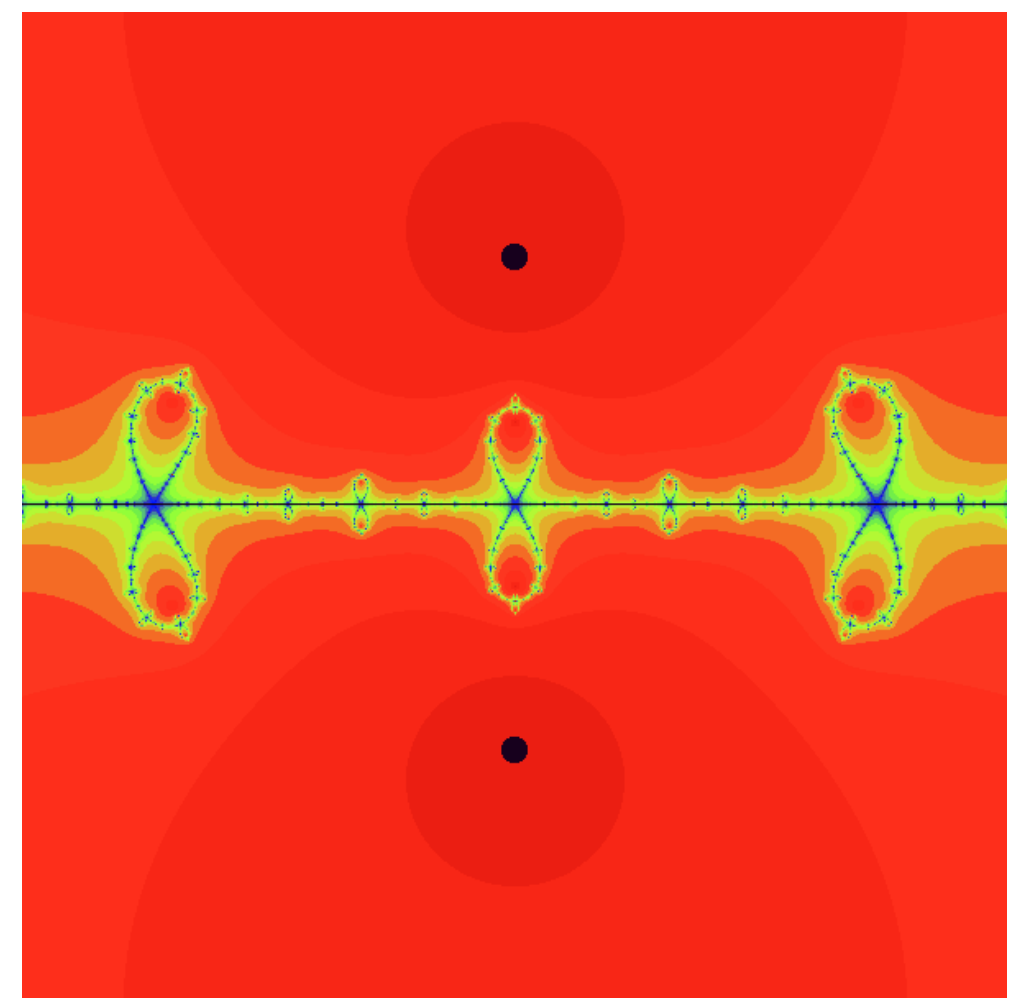

Fig. 1. Dynamical plane for the frozen derivative methods on quadratic polynomials

From Theorem 2, we know that Newton's iteration function on any quadratic polynomial is conjugated to $z^{2}$. In this case, we prove that $O_{p}$ on quadratic polynomials has a more complicated expression:

Theorem 6 Let $p(z)$ be a quadratic polynomial with distinct roots. The fixed 
point operator associated to the frozen derivative methods $O_{p}(z)$ has the following properties:

i) $O_{p}(z)$ is globally, analytically conjugate to the rational map $z^{3} \frac{z+2}{2 z+1}$.

ii) The dynamics of this operator implies that the unit circle $S^{1}(z)=\{z \in$ $\hat{\mathbb{C}}:|z|=1\}$ is included in the invariant Julia set. Moreover, this set is connected.

iii) The Fatou set is defined by the two basins of attraction of the superattracting fixed points: 0 and $\infty$.

Proof: As before, we consider the conjugacy map

$$
h(z)=\frac{z-i \sqrt{c}}{z+i \sqrt{c}}
$$

which has the same properties as in the previous results: $h(\infty)=1, h(i \sqrt{c})=$ $0, h(-i \sqrt{c})=\infty$. So,

$$
h^{-1}(z)=i \sqrt{c} \frac{z+1}{1-z}
$$

and, therefore

$$
B(z)=\left(h \circ O_{p} \circ h^{-1}\right)(z)=z^{3} \frac{z+2}{2 z+1}
$$

is a rational map of degree three that has superattracting fixed points at 0 and $\infty$. As in the previous case, this map does not depends on the parameter c. Moreover, for every $z \in S^{1}$, then $B(z) \in S^{1}$

$$
\forall z \in S^{1},|z|=1 \Rightarrow\left|z^{3} \frac{z+2}{2 z+1}\right|=\left|z^{3}\right|\left|\frac{z+2}{2 z+1}\right|=\left|\frac{z+2}{2 z+1}\right|
$$

and

$$
\left|\frac{z+2}{2 z+1}\right|=\left|\frac{(z+2)(2 \bar{z}+1)}{(2 z+1)(2 \bar{z}+1)}\right|=\left|\frac{z+4+4 \bar{z}}{5+4 \operatorname{Re}(z)}\right|=1
$$

since $|z+4+4 \bar{z}|=5+4 \operatorname{Re}(z)$.

Therefore, the unit circle is invariant under this function $B: S^{1} \rightarrow S^{1}$, and it separates the two basins of attraction of the two superattractor fixed points: 0 and $\infty$.

Because of the rational part, the Julia set is more complicated than the unit circle obtained in the Newton's method; nevertheless, all the points in the unit circle belongs to the Julia set (see Figure 2). As above, the Julia set separates the two basins of attraction of the two superattractor fixed points: 0 and $\infty$. 


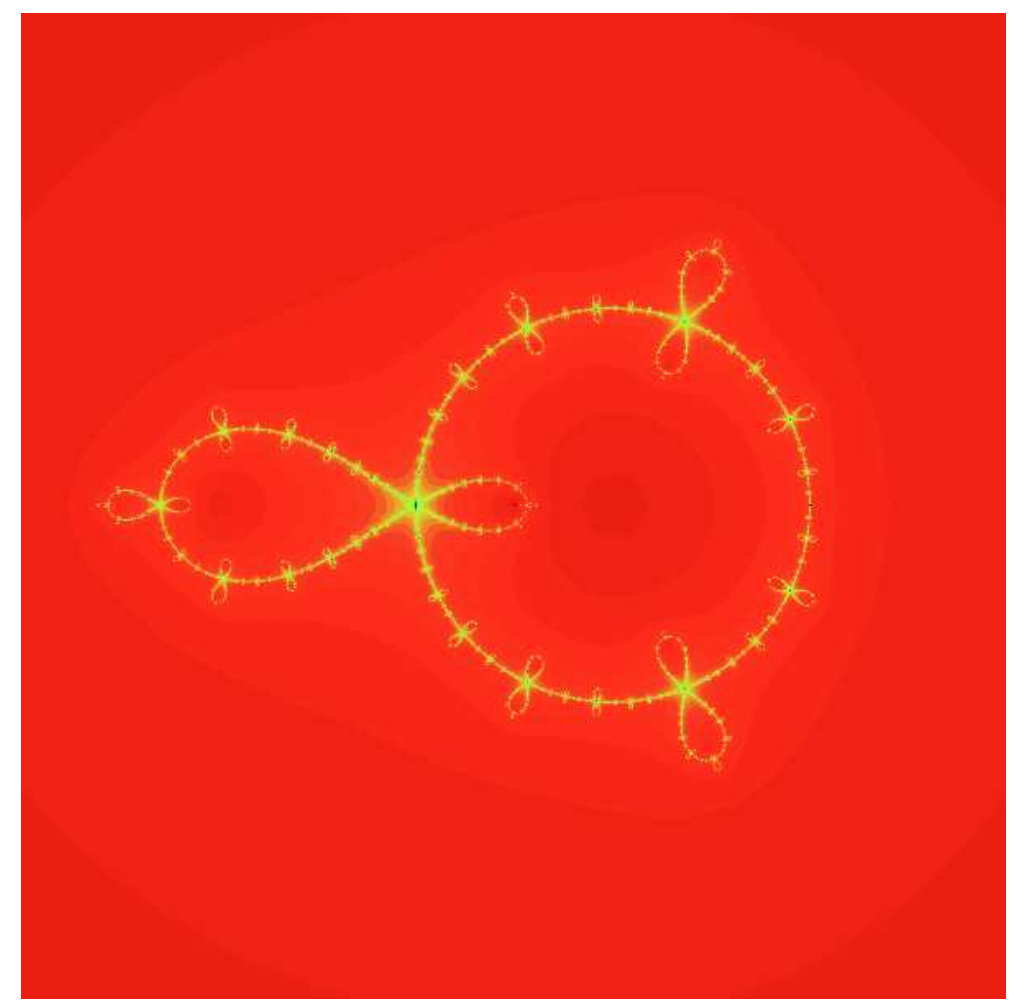

Fig. 2. Julia set for the frozen derivative methods on quadratic polynomials

\subsection{Iterative Methods coming from operator $O_{f}$}

There are some well known iterative methods that come from this operator. For example, the Potra-Pták's Method on the complex plane is a particular case of the previous method (15) for the case of $m=2, B_{j}=1, j=1,2$; $\tau_{1}=0, \tau_{2}=1$. The corresponding operator is:

$$
T_{f}(z)=z-\frac{f(z)}{f^{\prime}(z)}-\frac{f\left(z-\frac{f(z)}{f^{\prime}(z)}\right)}{f^{\prime}(z)} .
$$

There are two other methods, related with the golden ratio number, coming from the general formula (15), that are also of order three (see [15])

$$
\begin{aligned}
y_{k} & =x_{k}-q \frac{f\left(x_{k}\right)}{f^{\prime}\left(x_{k}\right)}, \\
x_{k+1} & =x_{k}-p_{1} \frac{f\left(y_{k}\right)}{f^{\prime}\left(x_{k}\right)},
\end{aligned}
$$

and 


$$
\begin{aligned}
y_{k} & =x_{k}+\frac{1}{q} \frac{f\left(x_{k}\right)}{f^{\prime}\left(x_{k}\right)}, \\
x_{k+1} & =x_{k}-p_{2} \frac{f\left(y_{k}\right)}{f^{\prime}\left(x_{k}\right)},
\end{aligned}
$$

where $p_{1}=\frac{3+\sqrt{5}}{2}, p_{2}=\frac{3-\sqrt{5}}{2}$ and $q=\frac{2}{1+\sqrt{5}}$. The corresponding operators are:

$$
\begin{aligned}
& O 1_{f}(z)=z-p_{1} \frac{f\left(z-q \frac{f(z)}{f^{\prime}(z)}\right)}{f^{\prime}(z)}, \\
& O 2_{f}(z)=z-p_{2} \frac{f\left(z+\frac{1}{q} \frac{f(z)}{f^{\prime}(z)}\right)}{f^{\prime}(z)} .
\end{aligned}
$$

As we have proved before, when we apply these three different methods on quadratic polynomial defined on the complex plane, $p(z)=z^{2}+c$, we obtain the same operator $O_{p}(z)=\frac{3 z^{4}-6 c z^{2}-c^{2}}{8 z^{3}}$. Therefore,

Remark The dynamics of these three known methods on quadratic polynomial defined on the complex plane, $p(z)=z^{2}+c, c \in \mathbb{C}$ are the same. The dynamical plane is shown in Figure 1.

\section{Conclusions}

Firstly, we would mention that we have obtained the conjugacy function of the operators associated to multi-points iteration methods and frozen derivative methods applied on quadratic polynomials.

Secondly, we note that the dynamical plane for the operator of the multi-points iteration methods on quadratic polynomials is the same than in the Newton case. This is explained from the fact that the two operators are conjugate to powers of $z$ (Theorems 2 and 4 ).

Finally, the Julia set in the dynamical plane for the operator of the frozen derivative methods is more complicated. Theorem 6 explain this difference because it shows that this operator is conjugated to the product of a monomial and a rational function.

Acknowledgments: The authors thank to Professors X. Jarque and A. Garijo for their help. 


\section{References}

[1] J. Curry, L. Garnet and D. Sullivan, On the iteration of a rational function: Computer experiments with Newton's method, Comm. Math. Phys., 911983 267-277.

[2] A. Douady and J.H. Hubbard, On the dynamics of polynomials-like mappings, Ann. Sci. Ec. Norm. Sup. (Paris), 18 (1985) 287-343.

[3] P. Blanchard, The Dynamics of Newton's Method, Proc. of Symposia in Applied Math., 49 (1994) 139-154.

[4] N. Fallega, Invariants en dinàmica complexa, Butlletí de la Soc. Cat. de Matemàtiques, 23(1) (2008) 29-51.

[5] S. Amat, C. Bermúdez, S. Busquier and S. Plaza, On the dynamics of the Euler iterative function, Applied Mathematics and Computation, 197 (2008) 725-732.

[6] S. Amat, S. Busquier and S. Plaza, A construction of attracting periodic orbits for some classical third-order iterative methods, J. of Computational and Applied Mathematics, 189 (2006) 22-33.

[7] J.M. Gutiérrez, M.A. Hernández and N. Romero, Dynamics of a new family of iterative processes for quadratic polynomials, J. of Computational and Applied Mathematics, 233 (2010) 2688-2695.

[8] S. Plaza and N. Romero, Attracting cycles for the relaxed Newton's method, J. of Computational and Applied Mathematics, 235 (2011) 3238-3244.

[9] S. Amat, S. Busquier and S. Plaza, Chaotic dynamics of a third-order Newtontype method, J. Math. Anal. Appl., 366 (2010) 24-32.

[10] F.A. Potra and V. Pták, Nondiscrete introduction and iterative processes, Research Notes in Mathematics, 103, Pitman, Boston, 1984.

[11] S. Plaza, Conjugacy classes of some numerical methods, Proyecciones, (2001) $1-17$.

[12] A. Cordero and J.R. Torregrosa, On interpolation variants of Newton's method for functions of several variables, J. of Computational and Applied Mathematics, 234 (2010) 34-43.

[13] M. Frontini and E. Sormani, Some variant of Newton's method with third-order convergence, Applied Mathematics and Computation, 140 (2003) 419-426.

[14] A.Y. Ozban, Some new variants of Newton's Method, Applied Mathematics Letters, 17 (2004) 677-682.

[15] A. Cordero, J.L. Hueso, E. Martínez and J.R. Torregrosa, Multi-Point Iterative Methods for Systems of Nonlinear Equations, Lecture notes in control and information sciences, 389 (2009) 259-267. 